\title{
ON A THEOREM OF AMBROSE
}

\author{
DAVID J. WRAITH \\ (Received 26 July 2004; revised 15 February 2005) \\ Communicated by C. Hodgson
}

\begin{abstract}
A Riccati inequality involving the Ricci curvature can be used to deduce many interesting results about the geometry and topology of manifolds. In this note we use it to present a short alternative proof to a theorem of Ambrose.
\end{abstract}

2000 Mathematics subject classification: primary 53C20.

\section{Introduction}

This paper concerns the Ricci curvature of a complete Riemannian manifold. The interaction between the Ricci curvature and the topology of a manifold, or between the Ricci curvature and other geometric phenomena is far from fully understood.

A smooth manifold can be equipped with many different Riemannian metrics, each of which gives the manifold geometry. One might ask what kind of curvatures a given manifold can display. In particular, one might look for geometries which are in some sense special. Important examples of 'special' geometries are those for which all curvatures are positive, or negative, or zero (flat).

There are three popular measures of curvature: the sectional, the Ricci, and the scalar. Sectional curvature is the strongest measure or curvature. Ricci is in some sense an average of sectional curvatures, and scalar an average of Ricci curvatures. Hence these curvatures contain successively less information about the bending of a manifold.

As noted above, we will focus on the Ricci curvature. It was established by Lohkamp [3] that in dimensions at least three, there are no topological obstructions

(C) 2006 Australian Mathematical Society $1446-7887 / 06 \$ \mathrm{~A} 2.00+0.00$ 
for a manifold to admit a complete metric with everywhere negative Ricci curvature. Therefore all manifolds of dimension at least three admit such metrics - even spheres! The question of which manifolds admit metrics of positive Ricci curvature is, in contrast, far from resolved. It is known, however, that there are topological obstructions. Most of these arise from the study of positive scalar curvature, see for example Stolz [6]. At the present time there is only one obstruction known which does not arise in this way. This originates in the classical theorem of Myers [4].

THEOREM 1.1. Suppose that $M^{n}$ is a complete Riemannian manifold for which the Ricci curvature satisfies

$$
\operatorname{Ric}(v, v) \geq(n-1) \delta>0
$$

for some constant $\delta$ and all unit tangent vectors $v$. Then $M$ is compact with diameter at most $\pi / \sqrt{\delta}$, and the fundamental group $\pi_{1} M$ is finite.

Myers' theorem is useful for getting a feel for the Ricci curvature. Very loosely speaking, it could be re-phrased by saying that the greater the Ricci curvature, the smaller the manifold.

Several variants of this result appear in the literature, see, for example, Sprouse [5]. We state one such variant, which was established by the author in [7].

THEOREM 1.2. Let $M^{n}$ be a complete, non-compact Riemannian manifold with nonnegative Ricci curvature and $\gamma(t), t \geq 0$, a unit speed ray in $M$. For $\delta>0$, let

$$
I_{\delta}=\left\{t>0: \operatorname{Ric}\left(\gamma^{\prime}(t), \gamma^{\prime}(t)\right) \geq(n-1) \delta\right\} .
$$

Then $I_{\delta}$ is Lebesgue measurable with $\mu\left(I_{\delta}\right) \leq \pi /(2 \sqrt{\delta})$.

The main idea in the proof of this result was to study the Riccati inequality for the mean curvature of distance spheres (see (1) in Section 2). A careful analysis of this simple inequality is all that is required. Myers' Theorem can also be deduced from the Riccati inequality, however it is more usually proved via the Calculus of Variations.

The main aim of this paper is to investigate a result of Ambrose [1], which can be viewed as another variant of Myers' Theorem.

THEOREM 1.3. Suppose there exists a point $p$ in a complete Riemannian manifold $M$ for which every geodesic $\gamma(t)$ emanating from $p$ satisfies

$$
\lim _{t \rightarrow \infty} \int_{0}^{t} \operatorname{Ric}\left(\gamma^{\prime}(u), \gamma^{\prime}(u)\right) d u=\infty
$$

Then $M$ is compact. 
Unlike the other results mentioned above, no assumption is made here about the sign of the Ricci curvature. Despite its simplicity and intuitive content, it is the author's experience that this result is not widely known.

Ambrose proves this using the Calculus of Variations. Our objective is to show that this result, like those of Myers [4] and Wraith [7], is actually inherent in the Riccati inequality. The alternative proof that we offer also has the advantage of being shorter than the original.

We will actually prove the following.

THEOREM 1.4. Let $\gamma(t), t \geq 0$, be a ray in a complete, non-compact Riemannian manifold. Then if

$$
\lim _{t \rightarrow \infty} \int_{0}^{t} \operatorname{Ric}\left(\gamma^{\prime}(u), \gamma^{\prime}(u)\right) d u
$$

exists, it must take a value less than infinity.

It is easy to see that this is equivalent to the Ambrose result, given that there is a ray emanating from every point in a complete non-compact manifold.

\section{Proof of Theorem 1.4}

Consider a point $p \in M^{n}$ and a unit speed ray $\gamma(t)$ issuing from $p$ (in other words $\gamma(0)=p)$. For every $t>0$, let $m(t)$ be the mean curvature of the distance sphere of radius $t$ about $p$ at the point $\gamma(t)$. Here we are assuming that the mean curvature is computed with respect to the inward unit normal. Note that $m(t)$ must be smooth for all $t>0$ along any ray, as the distance sphere is non-degenerate in a neighbourhood of $\gamma(t)$ for all $t>0$. It is well known that $m(t)$ satisfies a Riccati inequality:

$$
\operatorname{Ric}\left(\gamma^{\prime}(t), \gamma^{\prime}(t)\right) \leq-m^{\prime}(t)-\frac{1}{n-1} m^{2}(t)
$$

see Cheeger [2] for details.

Assume now that $\gamma(t)$ is a ray for which

$$
\lim _{t \rightarrow \infty} \int_{0}^{t} \operatorname{Ric}\left(\gamma^{\prime}(u), \gamma^{\prime}(u)\right) d u=\infty .
$$

We show that there exists a finite $t_{\infty}>0$ for which $\lim _{t \rightarrow t_{\infty}^{-}} m(t)=-\infty$, which contradicts the smoothness of $m(t)$ at all $t>0$.

For convenience, define $f(t)=-m(t)$. From the Riccati inequality we obtain

$$
\int_{1}^{t} \operatorname{Ric}\left(\gamma^{\prime}(u), \gamma^{\prime}(u) d u \leq \int_{1}^{t} f^{\prime}(u)-\frac{1}{n-1} f^{2}(u) d u .\right.
$$


Assuming (2), it follows that

$$
\lim _{t \rightarrow \infty} \int_{1}^{t} f^{\prime}(u)-\frac{1}{n-1} f^{2}(u) d u=\infty .
$$

We show the existence of a $t_{\infty}$ as above under the weaker condition

$$
\lim _{t \rightarrow \infty} f(t)-\int_{1}^{t} f^{2}(u) d u=\infty .
$$

An immediate consequence of this condition is that $\lim _{t \rightarrow \infty} f(t)=\infty$.

It is clear from (3) that there exists $t_{1}>1$ such that $f(t)-\int_{1}^{t} f^{2}(u) d u>10$ for all $t \geq t_{1}$. Inductively define $t_{n+1}=t_{n}+10^{1-n}$. Applying the inequality in the previous line we see that if $f(t) \geq k$ for $t \geq t_{n-1}$, then for all $t \geq t_{n}$,

$$
f(t)>\int_{t_{n-1}}^{t_{n}} f^{2}(u) d u \geq\left(t_{n}-t_{n-1}\right) k^{2} .
$$

It follows by induction that $f(t) \geq 10^{n}$ for $t \geq t_{n}$, hence

$$
\lim _{n \rightarrow \infty} f\left(t_{n}\right)=\lim _{x \rightarrow 10 / 9} f\left(t_{1}+x\right) \geq \lim _{n \rightarrow \infty} 10^{n} .
$$

Thus $t_{\infty} \leq t_{1}+10 / 9$, and we have the desired contradiction.

\section{Acknowledgements}

The author would like to thank Stephen Buckley for a valuable conversation.

\section{References}

[1] W. Ambrose, 'A theorem of Myers', Duke Math. J. 24 (1957), 345-348.

[2] J. Cheeger, Critical points of distance functions and applications to geometry, Lecture Notes in Math. 1504 (Springer, Berlin, 1991) pp. 1-38.

[3] J. Lohkamp, 'Metrics of negative Ricci curvature', Ann. of Math. (2) 140 (1994), 655-683.

[4] S. Myers, 'Riemannian manifolds with positive mean curvature', Duke Math. J. 8 (1941), 401-404.

[5] C. Sprouse, 'Integral curvature bounds and bounded diameter', Comm. Anal. Geom. 8 (2000), 531-543.

[6] S. Stolz, 'Simply connected manifolds of positive scalar curvature', Bull. Amer. Math. Soc. (N.S.) 23 (1990), 427-432.

[7] D. Wraith, 'Ricci curvature decay on open manifolds', Bull. London Math. Soc. 35 (2003), 72-78.

\section{Department of Mathematics}

National University of Ireland Maynooth

Ireland

e-mail: dwraith@maths.nuim.ie 